\title{
Suborbital Vehicles to Study Transition Adaptation to Spaceflight - Why Biologists Should Care About the New Suborbital Flight Opportunities
}

\author{
Robert J. Ferl
}

Department of Horticultural Sciences, Interdisciplinary Center for Biotechnology Research, University of Florida, Gainesville, FL 32610

\begin{abstract}
The advent of the new generation of suborbital space vehicles is opening up a new and exciting realm of space science that should be of great interest to biologists. These vehicles make it possible to explore biological responses and adaptations that occur in the first few minutes of entering spaceflight and also in the first few minutes after return from space. Historically these transition stages in spaceflight have simply not been available for research, especially within human-rated vehicles. Given that complex biological responses are seldom linear over time, and that essentially all current experiments on the International Space Station (ISS) are conducted after stabilization on orbit, biologists are missing the chance to understand the pathways that lead from terrestrial existence to successful spaceflight
\end{abstract}

Key words Suborbital Research; Spaceflight

Adaptation; Transition to Space; Arabidopsis

Correspondence to: Robert J. Ferl

Department of Horticultural Science and the Interdisciplinary Center for Biotechnology Research

University of Florida

Gainesville, FL 32610

Telephone: 352-273-8030

E-mail: robferl@ufl.edu adaptation and back. Studies conducted on suborbital spacecraft can therefore be an innovative approach to filling a substantial gap in knowledge regarding the temporal dynamics of biological responses to successful spaceflight physiological adaptation.

\section{INTRODUCTION}

One of the fundamental marvels of biology is its ability to adapt to changing and even new environments. This happens over evolutionary time through mutation and selection in populations and it happens in shorter timeframes through physiological adaptation within an individual. Physiological adaptation is guided at least in part by altered gene expression, and the changes in gene expression that accompany spaceflight physiological adaptation on the ISS are under current intense study. The scientific community has excelled in taking advantage of the Space Shuttle and ISS to characterize the different metabolic and gene expression states that exist between organisms on the ground and their orbital counterparts. The literature is greatly expanding with reports on the physiological differences that occur in many species between ISS and ground reference habitats.

But what is missing in all the current studies is any opportunity to characterize the transition states - the pathways that lead from terrestrial 
existence to spaceflight existence. What are the early responses to spaceflight and how do they compare to longer term responses of full adaptation? The purpose of this commentary is to discuss the case for caring about and approaching this area of research within the new suborbital vehicles, first by outlining a central hypothesis that there is much to be learned about spaceflight adaptation by understanding the transition from standing on the ground to floating in space, and then by developing some premise propositions and background that support the general approach toward addressing the hypothesis. This commentary is not meant as a comprehensive review of biological adaptation to spaceflight. Much of the specific information presented here is drawn from experience with plant spaceflight experiments, but given the fundamental principles that guide all biological responses, the arguments are very likely appropriate to all biology.

\section{CONSIDERING THE HYPOTHESIS}

The hypothesis that underpins an interest in suborbital vehicles can be stated something like this: All organisms undergo early physiological adaptation responses during the transition from Earth to spaceflight, responses that are different than the longer term, stable adaptation states that have so far been examined in orbital spaceflight experiments. The idea is that elucidating biological transitions among spaceflight phases is richly informative toward a comprehensive understanding of what it takes to go to, live in, and return from space. From a basic science perspective, this elucidation is also key to two related and important concepts. The first concept is that understanding the process of achieving an adaptive state provides a mechanistic understanding of that adaptive state. The second concept reaches beyond that singular adaptation and suggests that understanding spaceflight responses contributes to a fundamental understanding of how biology responds to environments that are beyond the evolutionary experience of the organism.

A main aspect of this hypothesis is that early responses to spaceflight are most likely quite different than the longer term adaptation responses that the community is now elucidating with experiments that are initiated and run on the ISS. For example, in the area of plant gene expression, to date all published molecular biology of plants in spaceflight has used samples that were in space for long periods of time (e.g. Correll et al., 2013; Paul et al., 2012b; Paul et al., 2013b) and therefore examine the long-term, stably adaptive state. And although there are transcriptome data from the intermittent microgravity of parabolic flight (Paul et al., 2011), no molecular studies have examined the launch and early adaptation phases to spaceflight or the return from spaceflight.

The baseline situation is that biologists have, as yet, had essentially no ability to conduct experiments on the early stages of transition from Earth to space - that is, from the resting state before launch to the early flight stages of being in space. Instead, most modern space plant biology experiments are initiated after the seeds are on orbit. Certainly all current molecular response datasets are derived from plants adapted to spaceflight for days. And experiments that did launch live seedlings were not sampled for molecular responses until long after orbit insertion - usually days. There is, therefore, a large gap in understanding regarding the physiological and molecular adaptations that occur as biology enters the spaceflight realm. More suborbital spaceflight vehicles will soon provide facile access to these first few minutes of spaceflight, creating unique potential to address this gap in knowledge and address the central hypothesis.

In accordance with the notion of addressing the "multiple stimuli encountered in spaceflight environments" (Decadal-SurveyCommittee, 2011), the use of suborbital vehicles would allow the examination of the complex biological responses within human-rated spaceflight vehicles undergoing transitions to and from spaceflight-boosted high-g launch, coast into suborbital space and microgravity for periods of 6-8 minutes, and then high- $g$ return for landing and recovery at $1 \mathrm{~g}$. And given that, at present, the human-rated suborbital vehicles are using the Mid Deck Locker (MDL) as the central organizing form factor, suborbital research can be comparable in instrumentation and complexity to ISS science, thereby more directly contributing to the comprehensive understanding of spaceflight physiological adaptation (Decadal-Survey-Committee, 2011). 
The Rationale for Suborbital Biology from a Molecular Perspective

Spaceflight responses

Physiological adaptation to spaceflight requires a molecular response that guides changes in metabolism and structure compared to being on the ground. Spaceflight apparently induces complex adaptive physiological responses that are recorded in the gene expression patterns in space compared to on the ground. Some - but not all - of these changes in gene expression are due to gravity, yet all are likely due to the experience of spaceflight (Decadal-Survey-Committee, 2011). This conclusion is now supported by a strong list of shuttle and ISS experiments, including experiments on Arabidopsis thaliana (arabidopsis) and other plants. Molecular genetic data joined spaceflight plant biology analyses as sample preservation and sample return capabilities allowed the recovery of high quality RNA. There are sets of published plant spaceflight genomewide expression data indicating there were a number of genes that showed moderate changes between flight and ground controls in arabidopsis (Paul et al., 2005). Yet in wheat grown in different hardware and germinated on orbit, there were virtually no statistically significant differences between the gene expression patterns of space-grown and ground control plants (Stutte et al., 2006). In the fern Ceratopteris richardii, over $5 \%$ of the genes examined from germinating spores were differentially expressed, a large contingent of which were associated with calcium-mediated processes (Salmi et al., 2011a; Salmi and Roux, 2008). Further advances in spaceflight operations and hardware, as well as advances in molecular analyses, have contributed to increasingly well-controlled whole transcriptome analyses of model plant systems such as arabidopsis (e.g.: Correll et al., 2013; Paul et al., 2012b; Paul et al., 2013b) and mizuna (Brassica rapa nipposinica) (Sugimoto et al., 2014). The transcriptome data are supported by other molecular data that suggest a microgravity environment influences rapid-response signaling such as auxin transport and mechanosensitive ion channels (Salmi et al., 2011b; Scherer and Pietrzyk, 2014), and can provide unique insights into cellular remodeling (Nakashima et al., 2014; Paul et al., 2012a), cell proliferation (Matia et al., 2010), hydrotropism (Takahashi, 2011), and phototropism (Kiss et al., 2012) outside the influence of gravity. Spaceflight requires complex adaptation (recently reviewed in: Paul et al., 2013a; Ruyters and Braun, 2014; Wolverton and Kiss, 2009; Wyatt and Kiss, 2013).

While such studies clearly document gene expression differentials between spaceflight and ground, all of these studies represent samples that have spent many days and in some cases their entire sporophytic life in the spaceflight environment. There are no studies on gene expression that probe the initial transition to spaceflight, other than some published data from parabolic flights (Aubry-Hivet et al., 2014; Hausmann et al., 2014; Paul et al., 2011)

Spaceflight data are elucidating the metabolic and structural changes required for life in space. Recent transcriptome work with arabidopsis shows that plants respond to spaceflight in an organ-specific, and cell-type-specific manner, suggesting there are adaptation requirements that are varied and specific. Leaves, hypocotyls, and roots each display unique patterns of response, yet many gene functions within the responses are related. Genes associated with cell architecture and growth hormone signaling are particularly prominent and may correlate with changes in morphology observed in spaceflight plants (Paul et al., 2013b). Although functionally related genes were differentially represented in leaves, hypocotyls, and roots, the expression of individual genes varied substantially across organ types, indicating that there is no single response to spaceflight. Rather, each organ employed its own response tactics within a shared strategy, largely involving cell wall architecture. Further, gene expression data suggest that in the absence of gravity plants rely on other environmental cues to initiate the morphological responses essential to successful growth and development, and that the basis for that reliance lies in the differential expression of genes in an organ-specific manner that apparently maximizes the utilization of these signals (Paul et al., 2013b). These trends are seen in younger plants as well - plants of only 4 days old showed differential expression of genes involved in regulating cell polarity (i.e., auxin, calcium, lipid metabolism), cellwall develop-ment, oxygen status, and cell defense or stress (Correll et al., 2013). 
The molecular responses of spaceflight adaptation are also revealed by the cell-specific expression patterns of reporter genes assayed by imaging methods (for plant examples see Paul et al., 2001; Paul et al., 2011; Paul et al., 2013b). Some of the most intriguing plant biology responses to spaceflight and gravity occur in specific cell types and in localized expression patterns that can be lost in large-scale gene expression studies that harvest entire organs. Cellspecific expression of Green Fluorescent Protein (GFP) and other reporter genes have shown dramatic relocalization of expression patterns in rapid response to changes in gravity and in changes to spaceflight.

\section{Comparable Complex Biological Responses}

Complex biological responses are not linear with time or intensity of stimuli, but rather are temporally dynamic with phases that involve transition genes that do not have roles in the final adaptive state. The body of knowledge from environmental stimuli suggests strongly that molecular adaptation responses to spaceflight will be a rich and complex set of transitions. There is extensive literature identifying differential gene expression at various times after the onset of an environmental stimulus, including gravity. The molecular responses to simple changes in temperature are briefly highlighted here as examples of how the final adaptive state is achieved by multiple transition phases. Temperature is a seemingly unitary stimulus, yet any change in temperature is followed by a series of transition states until full adaptation is accomplished. In a classic and often cited example, Fowler and Thomashow (2002) found $\sim 300$ genes that were affected by a shift of arabidopsis to lower temperature. Of the $\sim 200$ genes up regulated by cold, $\sim 150$ were transiently up regulated at some point during the adaptation process, while only $\sim 60$ were associated with the long-term adaptation. Similarly, of the $\sim 80$ genes down regulated by cold, half were transient while half were longterm response.

Such complex biological responses in gene activity can be monitored by reporter gene driven GFP detection, both for cell specificity as well as temporal resolution. In another example that is particularly relevant to cell and organ specificity, the transient activation of heat shock genes was studied by imaging of Heat Shock Protein (HSP)gene-driven constructs (Kast et al., 2013). HSP 17.6, a commonly thermoregulated gene, was found to be both transiently and spatially regulated. HSP 17.6 shows different quantitative upregulation profiles that vary over time, since the onset of thermal stress and also dependent upon the degree of thermal stress. The use of GFP to determine the location of gene expression responses is also elegantly demonstrated in that Kast et al. (2013) showed remarkable differential localization of HSP-GFP gene expression in the various cells of roots over the time of the response. Therefore, HSP 17.6 shows clear differences in quantitative and spatial expression patterns depending upon both time and temperature as plants adapt to a rise in temperature.

These two examples, taken from the classic and modern plant environmental response literature, show the common themes among biological responses to the environments. There are temporal stages that are not simply linear with intensity and common among all cells. Rather, there are sets of changes - sometimes many sets of changes - which occur early in the response that disappear later in the adaptive process. Moreover, those changes are dynamic among tissue and cell types adding a spatial dimension to join the temporal dimension. Our understanding of spaceflight adaptation currently enjoys essentially no temporal dimension and certainly nothing about the initial responses to changes in the spaceflight environment.

That said, there are several direct examples that strongly suggest that relevant responses are very likely to happen early in the spaceflight experience. Gravity and mechanical stimulation of arabidopsis produces molecular changes that have been recorded at two minutes after application of the stimulus (Kimbrough et al., 2004), and gene expression and protein alterations have been noted within arabidopsis cells within a single parabolic flight parabola (Hausmann et al., 2014). Parabolic flight maneuvers also produce changes in gene expression of human endothelial cells (Grosse et al., 2012). The spaceflight relevant stimulations and timeframes indicate that the suborbital flight realm will offer a rich set of informative biological responses. 


\section{Suborbital Spaceflight}

The development of human-rated suborbital space vehicles now allows, for the first time, sophisticated biology experiments to be conducted during the transitions to and from spaceflight. In contrast, SpaceX Dragon takes days to get to the ISS and has essentially no capabilities for active experimentation immediately after launch. The Space Shuttle carried active experiments during launch and orbital insertion, but there exist no gene expression samples from the first few minutes or even the first few hours after launch. The new suborbital vehicles from Virgin Galactic and XCOR (as well as Blue Origin and potentially others) are, however, specifically designed to fly to space while allowing a direct and active focus to all phases of transitioning from ground to space. Virgin Galactic SpaceShipTwo and XCOR Lynx are designed as human-rated vehicles that will launch, float through an arc in space for minutes, then return to a runway landing at the launch site. These vehicles are designed to carry experimenters as well as automated payloads. Moreover, the vehicles and procedures are designed to allow humans to conduct experiments through all phases of the flights. To date, access to these vehicles has been limited to technology maturation as part of the NASA Flight Opportunities Program for technology development.

Can we really use, for example, Virgin Galactic's SpaceShipTwo or XCOR's Lynx as vehicles for quality biological research? SpaceShipTwo and Lynx are designed as fully human-rated spaceships. The SpaceShipTwo cabin operates as a shirtsleeve environment, maintaining pressure and temperature for the benefit of human travelers, and as a result the internal environment of SpaceShipTwo is amenable to biological samples and to the operation of payloads by persons in flight. It was developed expressly for the conduct of suborbital spaceflight travel for paying passengers. Lynx is also a pressurized vehicle, though the pilot and passenger are expected to wear pressure suits. In recognition of their value as research platforms, both SpaceShipTwo and Lynx can accommodate racks such that standard MDL payloads can be flown along with researchers and/or flight test engineers.

The flight profile of SpaceShipTwo is intended to provide 3-4 minutes of quality microgravity. SpaceShipTwo takes off horizontally, mated to the White Knight Two mothership until launch altitude is reached. At launch, SpaceShipTwo is released, the rocket engine is fired, and the vertical ride at $\sim 5 g$ occurs until engine shutoff. SpaceShipTwo then floats through suborbital space before reentry and return to the spaceport runway for horizontal landing. The Lynx flight profile is similar but simpler. Lynx is intended to take off on its own, horizontally from a runway then pulling up to a near vertical high-g boost. The top of the
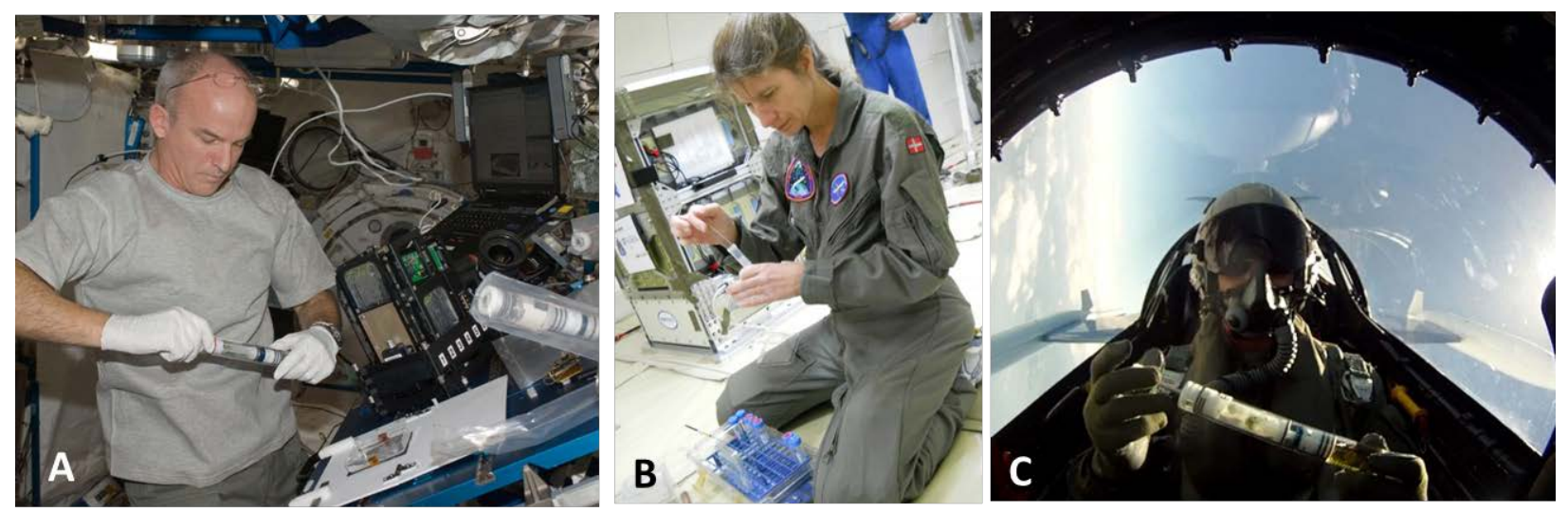

Figure 1. Operational molecular biology in spaceflight and spaceflight-related environments. (A) Astronaut Jeff Williams at the Multipurpose Work Area of the ISS, harvesting arabidopsis from our petri plates and fixing them in RNAlater in a KFT. (B) Anna-Lisa Paul harvesting during a NASA Flight Opportunities parabolic flight. (C) Robert Ferl activating KFT during F104 flight. 
Lynx arc is intended to provide 2-4 minutes of quality microgravity. It should be noted that these profiles are notional and should be considered approximate until active flight operations define actual profiles that will be available to science

Are there accepted operational methods to capture biological responses and particularly gene expression data from within these spaceflight vehicles? There is certainly precedent to say that the answer is very much "Yes." Published biological experiments and gene expression studies have been conducted in a wide range of vehicle platforms. Plant and other biology culture methods based on standard laboratory protocols exist for facile transport and handling of samples within vehicles, and harvesting plants and other samples in various spaceflight and related environments is well established. For molecular biology as a measure of spaceflight responses, the use of RNAlater is common practice in spaceflight scenarios, with KSC Fixation Tubes (KFTs) as the method of safely placing the plants in the preservative (Ferl et al., 2011). Successful plant harvests and subsequent molecular analyses have been conducted by astronauts in orbit and by scientists in parabolic and high performance aircraft (Figure 1). Given the design and concepts of operations described for SpaceShipTwo and Lynx, it is very likely that human-tended biological sample collection of various types should be very possible in suborbital flights. Moreover, there are many payload systems for biological experimentation that are designed as MDL experiments. Any instrument or experiment system that is capable of functioning within a powered MDL should be readily adaptable to the suborbital ships discussed above and others that may come on line.

\section{CONCLUSION}

The rationale for this commentary concludes along the following lines: like most biological responses to environmental conditions, physiological adaptation to spaceflight is very likely a complex molecular process that begins with early adaptive responses that transition to the different longer term responses that have so far been examined in spaceflight materials from shuttle and ISS, and the vehicles now exist to conduct experiments that elucidate the responses of those first few minutes of spaceflight adaptation. Based on that rationale, biologists should be aware of and interested in the opportunities that will exist in the near future, opportunities for cheaper multiple flights to space that provide access to experiments during the transition from Earth to space, and back.

\section{ACKNOWLEDGEMENTS}

This commentary was prepared to summarize a presentation by the author at the Suborbital Symposium at the 2013 meeting of the American Society for Gravitational and Space Research. As such, the content reflects the views from the perspective of the author and is not meant as a complete scientific review of the literature. Any errors in content are the sole responsibility of the author, who apologizes in advance for any misrepresentations which are surely unintended. Support for spaceflight and suborbital research was provided by NNX12AN69G, NNX12AK80G to Robert J. Ferl and Anna-Lisa Paul, and flights awarded by the NASA Flight Opportunities Program.

\section{REFERENCES}

Aubry-Hivet D, Nziengui H, Rapp K, Oliveira O, Paponov IA, Li Y, Hauslage J, Vagt N, Braun M, Ditengou FA, Dovzhenko A, Palme K (2014) Analysis of gene expression during parabolic flights reveals distinct early gravity responses in Arabidopsis roots. Plant Biology 16 Suppl 1: 129-141

Correll MJ, Pyle TP, Millar KD, Sun Y, Yao J, Edelmann RE, Kiss JZ (2013) Transcriptome analyses of Arabidopsis thaliana seedlings grown in space: implications for gravityresponsive genes. Planta 238(3): 519-533

Decadal-Survey-Committee (2011) Recapturing a Future for Space Exploration: Life and Physical Sciences Research for a New Era. Washington, D.C.: The National Academies Press

Ferl RJ, Zupanska A, Spinale A, Reed D, Manning-Roach S, Guerra G, Cox DR, Paul A-L (2011) The performance of KSC Fixation Tubes with RNAlater for orbital experiments: A case study in ISS operations for molecular biology. Advances in Space Research 48(1): 199-206 
Fowler S, Thomashow MF (2002) Arabidopsis transcriptome profiling indicates that multiple regulatory pathways are activated during cold acclimation in addition to the CBF cold response pathway. Plant Cell 14(8): $1675-1690$

Grosse J, Wehland M, Pietsch J, Ma X, Ulbrich C, Schulz H, Saar K, Hubner N, Hauslage J, Hemmersbach R, Braun M, van Loon J, Vagt $\mathrm{N}$, Infanger $\mathrm{M}$, Eilles C, Egli M, Richter P, Baltz T, Einspanier R, Sharbati S, Grimm D (2012) Short-term weightlessness produced by parabolic flight maneuvers altered gene expression patterns in human endothelial cells. FASEB Journal 26: 639-655

Hausmann N, Fengler S, Hennig A, FranzWachtel M, Hampp R, Neef M (2014) Cytosolic calcium, hydrogen peroxide and related gene expression and protein modulation in Arabidopsis thaliana cell cultures respond immediately to altered gravitation: parabolic flight data. Plant Biology 16 Suppl 1: 120-128

Kast EJ, Nguyen MD, Lawrence RE, Rabeler C, Kaplinsky NJ (2013) The RootScope: a simple high-throughput screening system for quantitating gene expression dynamics in plant roots. BMC Plant Biology 13: 158

Kimbrough JM, Salinas-Mondragon R, Boss WF, Brown CS, Sederoff HW (2004) The fast and transient transcriptional network of gravity and mechanical stimulation in the Arabidopsis root apex. Plant Physiology 136: 2790-2805

Kiss JZ, Millar KD, Edelmann RE (2012) Phototropism of Arabidopsis thaliana in microgravity and fractional gravity on the International Space Station. Planta 236(2): 635-645

Matia I, González-Camacho F, Herranz R, Kiss JZ, Gasset G, van Loon JJ, Marco R, Medina FJ (2010) Plant cell proliferation and growth are altered by microgravity conditions in spaceflight. Journal of Plant Physiology 167(3): 184-193

Nakashima J, Liao F, Sparks JA, Tang Y, Blancaflor EB (2014) The actin cytoskeleton is a suppressor of the endogenous skewing behaviour of Arabidopsis primary roots in microgravity. Plant Biology 16 Suppl 1: $142-150$
Paul A-L, Amalfitano CE, Ferl RJ (2012a) Plant growth strategies are remodeled by spaceflight. BioMed Central Plant Biology 12: 232

Paul A-L, Daugherty CJ, Bihn EA, Chapman DK, Norwood KL, Ferl RJ (2001) Transgene expression patterns indicate that spaceflight affects stress signal perception and transduction in Arabidopsis. Plant Physiology 126(2): 613-621

Paul A-L, Manak MS, Mayfield JD, Reyes MF, Gurley WB, Ferl RJ (2011) Parabolic flight induces changes in gene expression patterns in Arabidopsis thaliana. Astrobiology 11(8): 743-758

Paul A-L, Popp MP, Gurley WB, Guy CL, Norwood KL, Ferl RJ (2005) Arabidopsis gene expression patterns are altered during spaceflight. Advances in Space Research 36(7): 1175-1181

Paul A-L, Wheeler RM, Levine HG, Ferl RJ (2013a) Fundamental plant biology enabled by the space shuttle. American Journal of Botany 100(1): 226-234

Paul A-L, Zupanska A, Ostrow DT, Zhang Y, Sun $\mathrm{Y}$, Li JL, Shanker S, Farmerie WG, Amalfitano CE, Ferl RJ (2012b) Spaceflight transcriptomes: unique responses to a novel environment. Astrobiology 12(1): 40-56

Paul A-L, Zupanska AK, Schultz ER, Ferl RJ (2013b) Organ-specific remodeling of the Arabidopsis transcriptome in response to spaceflight. BioMed Central Plant Biology 13: 112

Ruyters G, Braun M (2014) Plant biology in space: recent accomplishments and recommendations for future research. Plant Biology 16 Suppl 1: 4-11

Salmi ML, Bushart TJ, Roux SJ (2011a) Autonomous gravity perception and responses of single plant cells. Gravitational and Space Biology 25(1): 6-13

Salmi ML, Roux SJ (2008) Gene expression changes induced by spaceflight in singlecells of the fern Ceratopteris richardii. Planta 229(1): 151-159

Salmi ML, ul Haque A, Bushart TJ, Stout SC, Roux SJ, Porterfield DM (2011b) Changes in gravity rapidly alter the magnitude and direction of a cellular calcium current. Planta 233(5): 911-920 
Scherer GFE, Pietrzyk P (2014) Gravitydependent differentiation and root coils in Arabidopsis thaliana wild type and phospholipase-A-I knockdown mutant grown on the International Space Station. Plant Biology 16 Suppl 1: 97-106

Stutte GW, Monje O, Hatfield RD, Paul A-L, Ferl RJ, Simone CG (2006) Microgravity effects on leaf morphology, cell structure, carbon metabolism, and mRNA expression of dwarf wheat. Planta 224: 1038-1049

Sugimoto M, Oono Y, Gusev O, Matsumoto T, Yazawa T, Levinskikh MA, Sychev VN, Bingham GE, Wheeler R, Hummerick M (2014) Genome-wide expression analysis of reactive oxygen species gene network in
Mizuna plants grown in long-term spaceflight. BioMed Central Plant Biology 14: 4

Takahashi H (2011) Hydrotropism and auxininducible gene expression in roots grown under microgravity conditions. In NASA ISS Payload Fact Sheet. Retrieved from http://www.nasa.gov/mission_pages/station/r esearch/experiments/766.html; (Accessed $11 / 25 / 14)$

Wolverton C, Kiss JZ (2009) An update on plant space biology. Gravitational and Space Biology 22: 13-20

Wyatt SE, Kiss JZ (2013) Plant tropisms: From Darwin to the International Space Station. American Journal of Botany 100: 1-3 